\title{
The Dynamics of Pendulums on Surfaces of Constant Curvature
}

\author{
P. Coulton*, R. Foote ${ }^{\dagger}$ G. Galperin ${ }^{\ddagger}$ \\ Department of Mathematics, \\ Eastern Illinois University and Wabash College
}

\begin{abstract}
In [1], the first and third authors investigated the motion of barbells on surfaces of constant curvature. It is natural to extend this study to pendulums.

We define the notion of a pendulum on a surface of constant curvature and study the motion of a mass at a fixed distance from a pivot. We consider some special cases for the pendulum.

Case 1: a pivot that moves with constant speed along a fixed geodesic.

Case 2: a pivot that undergoes acceleration along a fixed geodesic.

AMS classification: $37 \mathrm{~J}, 53 \mathrm{~A}, 70 \mathrm{E}, 85$.
\end{abstract}

Keywords: dynamical systems, geodesic, constant curvature, pendulum.

\footnotetext{
${ }^{*}$ Corresponding author, E-mail: cfprc@eiu.edu

${ }^{\dagger} \mathrm{E}$-mail: footer@wabash.edu

†E-mail: cfgg@eiu.edu
} 


\section{Introduction}

Consider a moving particle on a surface. An unconstrained particle would, of course, move along a geodesic with constant speed. The point of our work is to investigate the motion of a particle constrained to be at a constant distance from a point with a given motion.

A pendulum problem on a surface of constant curvature is defined as a pivot point and a mass connected to the pivot by a rigid massless rod of fixed length $\delta$. Assume that the pivot is constrained to move along some fixed curve with prescribed motion. The rod provides the only force on the mass in order to keep the mass a fixed distance from the pivot. No torque is applied to the rod, and so the acceleration of the mass is always in the direction of the rod.

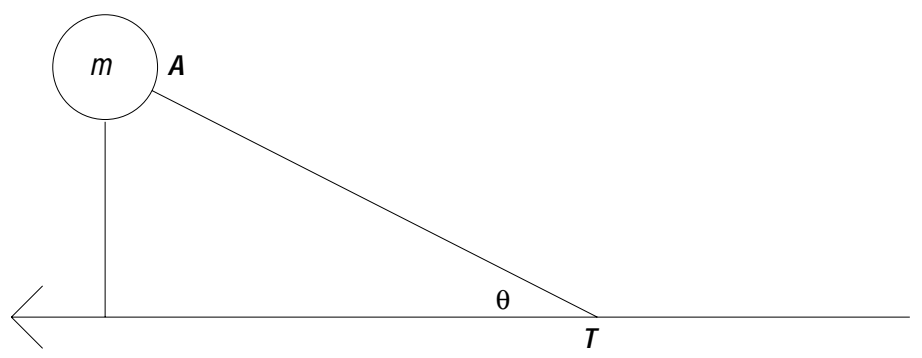

Figure 1. The motion of a pendulum

Figure 1 above illustrates the motion of a pendulum. The mass $m$ is at $A$ and the pivot is at $T$. The rigid rod is segment $A T$. Our results concern the case in which the pivot moves along a geodesic. We let $\theta$ denote the angle between the rod and the geodesic.

We shall prove the following:

Theorem A. Assume that the pivot of a pendulum moves with constant speed $v$ along a geodesic on a surface with constant curvature $K$. Let $\theta(t)$ be the angle that the rigid rod makes at time $t$ with respect to the direction of pivot motion. Then the pendulum satisfies the non-linear differential equation

$$
\frac{d^{2} \theta}{d t^{2}}=-v^{2} K \sin (\theta) \cos (\theta)
$$

The solution is periodic. Moreover, the period is approximately $2 \pi / v \sqrt{|K|}$ when $\theta$ is sufficiently close to a stable equilibrium for all $t$. If $K>0$, then 
there are stable equilibria at $\theta=0$ and $\theta=\pi$ and unstable equilibria at $\theta=\pi / 2$ and $\theta=3 \pi / 2$. If $K<0$, then there are unstable equilibria at $\theta=0$ and $\theta=\pi$ and stable equilibria at $\theta=\pi / 2$ and $\theta=3 \pi / 2$.

This differential equation indicates that the dynamics of a pendulum on a curved surface are sensitive to the absolute motion of the pivot, in contrast to the Euclidean case. It is somewhat surprising that the length of the pendulum does not play a role. Reparameterizing by the distance $s=v t$ traveled by the pivot, the differential equation can be rewritten as

$$
\frac{d^{2} \theta}{d s^{2}}=-K \sin (\theta) \cos (\theta)
$$

This shows that the dynamics of the pendulum do not depend on the magnitude of the speed of the pivot, but rather on the distance traveled by the pivot and the fact that its speed is constant. From this perspective, the period of the motion should be thought of as a distance, as opposed to a time. For small-amplitude oscillations sufficiently close to a stable equilibrium, the period is approximately $2 \pi / \sqrt{|K|}$. On a sphere, this is the circumference, and it follows that the mass of the pendulum is approximately following a geodesic slightly offset from the geodesic path of the pivot, that is, it approximately follows a path it would take were it not connected to the pivot. On a hyperbolic plane, the period is a natural distance identified by the pendulum dynamics. In contrast to the spherical case, the mass does not follow an approximate geodesic. Instead, it oscillates about a constant distance curve.

Theorem B. Assume that the pivot of a pendulum moves with constant speed $v$ along a geodesic on a surface with constant curvature $K$. Let $\theta(t)$ be the angle that the rigid rod makes at time $t$ with respect to the direction of pivot motion. If the pendulum starts at a stable equilibrium with initial intrinsic angular speed $d \theta /\left.d t\right|_{t=0}=v \sqrt{|K|}$, then it monotonically and asymptotically approaches an unstable equilibrium as $t \rightarrow \infty$. More specifically, if

$$
K>0, \quad \theta(0)=0, \quad \text { and }\left.\quad \frac{d \theta}{d t}\right|_{t=0}=v \sqrt{K}
$$

then $\theta$ is increasing and

$$
\lim _{t \rightarrow \infty} \theta(t)=\frac{\pi}{2} .
$$


Similarly, if

$$
K<0, \quad \theta(0)=\frac{\pi}{2}, \quad \text { and }\left.\quad \frac{d \theta}{d t}\right|_{t=0}=v \sqrt{-K},
$$

then $\theta$ is increasing and

$$
\lim _{t \rightarrow \infty} \theta(t)=\pi .
$$

It is interesting to note that when $K>0$ and the motion takes place on a sphere of radius $R=1 / \sqrt{K}$ in $\mathbb{R}^{3}$, the initial intrinsic angular speed in Theorem $\mathrm{C}$ is equal to the constant extrinsic angular speed of the pivot: $v \sqrt{K}=v / R=\omega$.

As a consequence of the proofs of Theorems A and B, we derive a conservation law for pendulum motion. We also consider the dynamics of the pendulum when the pivot is allowed to accelerate.

A barbell in a space of constant curvature $K$ consists of a pair of masses joined by a massless rigid rod of fixed length $d$. We say that the barbell is balanced if the masses are equal. Consider motion of a balanced barbell such that its center of mass moves along a geodesic. Note that the motion of a pendulum can also be considered as the motion of half of a balanced barbell.

If the entire motion of the barbell is along the geodesic, we say that it moves with a direct-motion. When the balanced barbell moves such that the rod is at a right angle to the direction of motion, we shall refer to the motion as a right-motion. If the direction of the rod from the center of mass to the leading mass makes an angle $\theta$ with respect to the geodesic of motion, we shall refer to the motion of the barbell as skew-motion, where $\theta \neq 0, \pi / 2$. In earlier work [1], the first and third authors showed that the direct-motion and right-motion of a balanced barbell are free (unconstrained) motions.
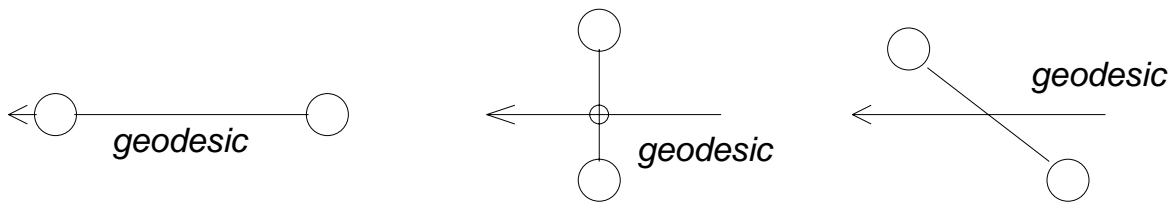

Figure 2. a) direct-motion, b) right-motion, c) skew-motion 
Theorem C If the midpoint of a balanced barbell moves along a geodesic on a surface of constant curvature with constant speed, then the motion of the individual masses is symmetric and identical to the motion of the equivalent pendulum problem.

Theorem D Assume that the pivot of a pendulum moves with speed $v(t)$ along a geodesic on a surface with constant curvature $K$. Let $\theta(t)$ be the angle that the rigid rod makes with the direction of the motion of the pivot. Then the pendulum satisfies the differential equation

$$
\frac{d^{2} \theta}{d t^{2}}=-K v(t)^{2} \cos \theta(t) \sin \theta(t)+\sqrt{|K|} a(t) \cot _{K} \delta \sin \theta(t),
$$

where $a(t)=d v / d t$ is the scalar acceleration. This has equilibria at $\theta=0, \pi$. They are stable when $K>0$ and unstable when $K<0$.

We analyze the small-amplitude oscillations in the special case when $v(t)=v_{0}+a t$.

We assume the following convention for spaces of constant curvature $K$ : if $K>0$,

$$
\cos _{K}(s)=\cos (s \sqrt{K}), \quad \sin _{K}(s)=\sin (s \sqrt{K}), \quad \tan _{K}(s)=\tan (s \sqrt{K})
$$

and if $K<0$, then

$$
\begin{gathered}
\cos _{K}(s)=\cosh (s \sqrt{-K}), \quad \sin _{K}(s)=\sinh (s \sqrt{-K}), \\
\tan _{K}(s)=\tanh (s \sqrt{-K}) .
\end{gathered}
$$

\section{Pendulum Motion for Constant Speed Pivots}

Figure 3 below illustrates additional quantities which will be used in the proof of Theorem A. We let $b$ denote the distance from $m$ to the geodesic and we let $a$ denote the distance from the pivot $T$ to the point $D$. Let $\delta$ be the distance from the pivot to the mass, i.e., the length of the rod. We observe that the geodesic triangle $\triangle A D T$ is a right triangle with right angle at $D$. We let $\alpha$ be the angle at $A$. 


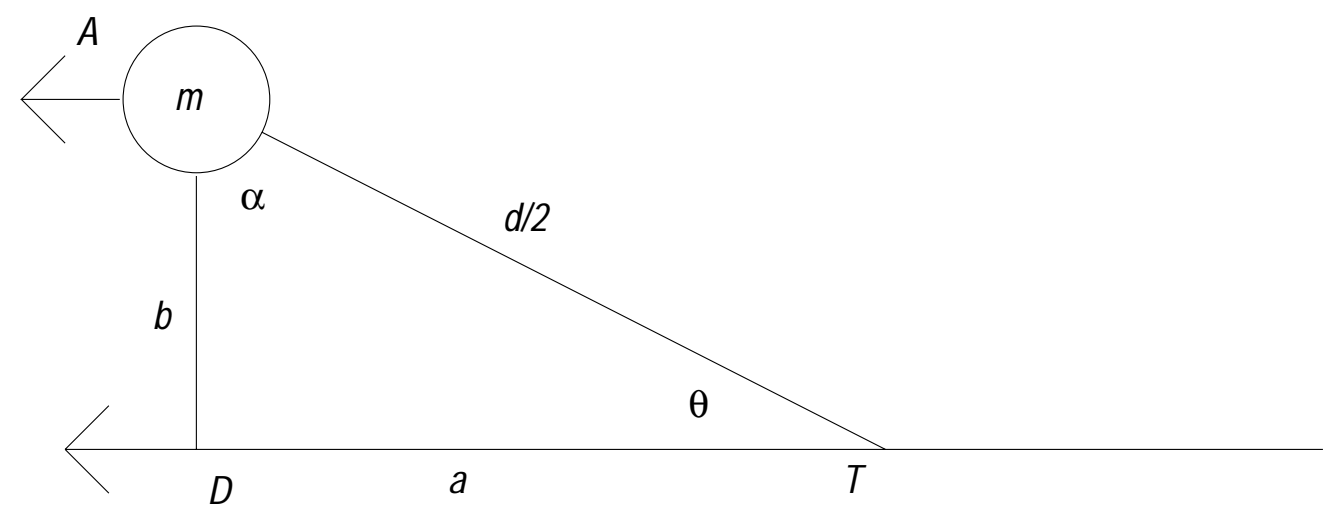

Figure 3. Skew-motion of a pendulum

\section{Proof of Theorem A.}

Case I: $K>0$. Assume that the pendulum pivot moves with constant speed along a geodesic on a surface of constant positive curvature. Let $\delta$ be the length of the rigid rod. We assume that $\delta<\pi R$, where $R=1 / \sqrt{K}$, and take the motion to be on the sphere $x^{2}+y^{2}+z^{2}=R^{2}$ in $\mathbb{R}^{3}$. The angular speed of the pivot is $\omega=v / R=v \sqrt{K}$, and the location of the pivot can be taken to be $R(\cos (\omega t) \hat{\mathbf{i}}+\sin (\omega t) \hat{\mathbf{j}})$. Letting $\theta$ be the angle between the direction of motion of the pivot and the rigid rod, the possible locations of the mass are parameterized by

$$
\begin{gathered}
F(t, \theta)=R \sin _{K} \delta[\sin \theta \hat{\mathbf{k}}+\cos \theta(-\sin (\omega t) \hat{\mathbf{i}}+\cos (\omega t) \hat{\mathbf{j}})] \\
+R \cos _{K} \delta(\cos (\omega t) \hat{\mathbf{i}}+\sin (\omega t) \hat{\mathbf{j}}) .
\end{gathered}
$$

For a particular motion of the mass we have $\theta=\theta(t)$, and the motion of the mass is $\sigma(t)=F(t, \theta(t))$, or

$$
\begin{gathered}
\sigma(t)=R \sin _{K} \delta[\sin (\theta(t)) \hat{\mathbf{k}}+\cos (\theta(t))(-\sin (\omega t) \hat{\mathbf{i}}+\cos (\omega t) \hat{\mathbf{j}})] \\
+R \cos _{K} \delta(\cos (\omega t) \hat{\mathbf{i}}+\sin (\omega t) \hat{\mathbf{j}}) .
\end{gathered}
$$

The acceleration in $\mathbb{R}^{3}$ is then 


$$
\begin{gathered}
\frac{d^{2} \sigma}{d t^{2}}=-\left[\frac{d \theta}{d t}\right]^{2} R \sin _{K} \delta[\sin (\theta(t)) \hat{\mathbf{k}}+\cos (\theta(t))(-\sin (\omega t) \hat{\mathbf{i}}+\cos (\omega t) \hat{\mathbf{j}})]+ \\
2\left[\frac{d \theta}{d t}\right] \omega R \sin _{K} \delta \sin (\theta(t))(\cos (\omega t) \hat{\mathbf{i}}+\sin (\omega t) \hat{\mathbf{j}})- \\
\omega^{2} R \sin _{K} \delta \cos (\theta(t))(-\sin (\omega t) \hat{\mathbf{i}}+\cos (\omega t) \hat{\mathbf{j}})- \\
\omega^{2} R \cos K \delta(\cos (\omega t) \hat{\mathbf{i}}+\sin (\omega t) \hat{\mathbf{j}})+ \\
{\left[\frac{d^{2} \theta}{d t^{2}}\right] R \sin _{K} \delta[\cos (\theta(t)) \hat{\mathbf{k}}-\sin (\theta(t))(-\sin (\omega t) \hat{\mathbf{i}}+\cos (\omega t) \hat{\mathbf{j}})]}
\end{gathered}
$$

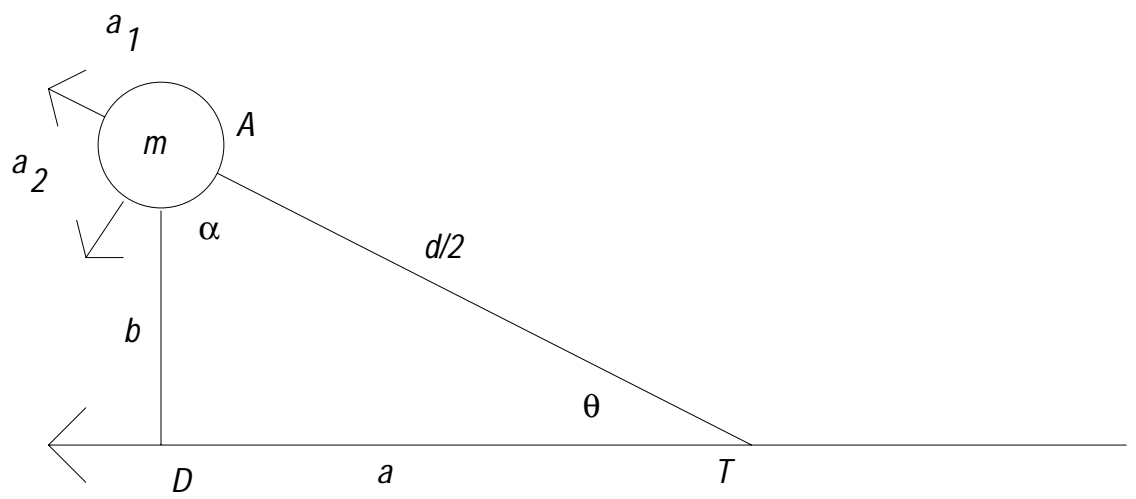

Figure 4. A standard frame

Let $\left\{\mathbf{a}_{1}, \mathbf{a}_{2}\right\}$ be an oriented, orthonormal frame for the tangent space of the sphere at the point $A$ (the location of the mass) such that $\mathbf{a}_{2}$ is perpendicular to the rigid rod at $A$. For a general location of the mass we have $\mathbf{a}_{2}=\frac{\partial F}{\partial \theta} /\left\|\frac{\partial F}{\partial \theta}\right\|$, which for the mass at location $\sigma(t)$ becomes

$$
\mathbf{a}_{2}(t)=\cos (\theta(t)) \hat{\mathbf{k}}-\sin (\theta(t))(-\sin (\omega t) \hat{\mathbf{i}}+\cos (\omega t) \hat{\mathbf{j}}) .
$$

If $g($,$) denotes the metric on the tangent space of the sphere, then$ the component of $d^{2} \sigma / d t^{2}$ tangent to the sphere and normal to the rod is $g\left(\frac{d^{2} \sigma}{d t^{2}}, \mathbf{a}_{2}(t)\right)$, which simplifies to

$$
g\left(\frac{d^{2} \sigma}{d t^{2}}, \mathbf{a}_{2}(t)\right)=R \sin _{K} \delta\left(\left[\frac{d^{2} \theta}{d t^{2}}\right]+\omega^{2} \sin (\theta(t)) \cos (\theta(t))\right) .
$$


Since the intrinsic force on the mass is applied only along the rigid rod, $g\left(\frac{d^{2} \sigma}{d t^{2}}, \mathbf{a}_{2}(t)\right)=0$, which yields the differential equation

$$
\frac{d^{2} \theta}{d t^{2}}=-\omega^{2} \sin (\theta(t)) \cos (\theta(t))=-\frac{K v^{2}}{2} \sin (2 \theta(t)) .
$$

Note that $\theta=0, \pi / 2, \pi, 3 \pi / 2$ are equilibrium solutions. Making the substitution $u=2 \theta$, the equation becomes

$$
\frac{d^{2} u}{d t^{2}}=-K v^{2} \sin u
$$

This is the equation of a planar pendulum in Euclidean space subject to a constant gravitational field, which is known to be periodic using energy considerations. The small-amplitude period is $2 \pi / v \sqrt{K}$. It has a stable equilibrium at $u=0 \bmod 2 \pi$, corresponding to $\theta=0, \pi$. Similarly, It has an unstable equilibrium at $u=\pi \bmod 2 \pi$, corresponding to $\theta=\pi / 2,3 \pi / 2$.

Case II: $K<0$. For simplicity, we assume the motion takes place in the hyperbolic plane of curvature $K$. We use the hyperboloid model in $\mathbb{R}^{3}$,

$$
x^{2}+y^{2}-z^{2}=\frac{1}{K}, \quad z>0,
$$

which inherits its geometry from the Minkowski inner product

$$
g((a, b, c),(x, y, z))=a x+b y-c z .
$$

Similar to Case I, set $R=1 / \sqrt{-K}$ and $\omega=v / R=v \sqrt{-K}$, and assume the pivot moves along the geodesic $R(\sinh (\omega t) \hat{\mathbf{i}}+\cosh (\omega t) \hat{\mathbf{k}})$. The circle of radius $\delta$ about the origin of the model is parameterized by

$$
c(\theta)=R \sin _{K} \delta(\cos \theta \hat{\mathbf{i}}+\sin \theta \hat{\mathbf{j}})+R \cos _{K} \delta \hat{\mathbf{k}} .
$$

Translating this circle to one centered at the pivot by means of the Minkowski isometry with matrix

$$
A(t)=\left(\begin{array}{ccc}
\cosh (\omega t) & 0 & \sinh (\omega t) \\
0 & 1 & 0 \\
\sinh (\omega t) & 0 & \cosh (\omega t)
\end{array}\right)
$$

yields the general position of the mass: 


$$
\begin{aligned}
F(t, \theta)=A(t) c(\theta)= & R \sin _{K} \delta[\sin \theta \hat{\mathbf{j}}+\cos \theta(\sinh (\omega t) \hat{\mathbf{k}}+\cosh (\omega t) \hat{\mathbf{i}})] \\
& +R \cos _{K} \delta(\cosh (\omega t) \hat{\mathbf{k}}+\sinh (\omega t) \hat{\mathbf{i}}) .
\end{aligned}
$$

From this point the proof is nearly identical to that of Case I with the substitution of hyperbolic functions for circular ones in the appropriate places, the permutation of the basis vectors, and the use of the Minkowski inner product.

The reversal of the stable and unstable equilibria result from the change in sign of $K$. More specifically, consider the substitution $u=2 \theta+\pi$. This changes the differential equation to

$$
\frac{d^{2} u}{d t^{2}}=K v^{2} \sin u=-|K| v^{2} \sin u
$$

and the reasoning can proceed as in Case I.

\section{Proof of Theorem B.}

By Theorem A, $\theta$ satisfies

$$
\frac{d^{2} \theta}{d t^{2}}=-K v^{2} \sin \theta \cos \theta=-\frac{K v^{2}}{2} \sin 2 \theta
$$

If $K>0$, let $u=2 \theta$. If $K<0$, let $u=2 \theta+\pi$. In both cases the equation becomes

$$
\frac{d^{2} u}{d t^{2}}=-|K| v^{2} \sin u
$$

which is the equation of a planar pendulum of length 1 in Euclidean space subject to a constant gravitational field of magnitude $g=|K| v^{2}$. Its stable and unstable equilibria at $u=0$ and $u=\pi$ correspond to the two stable and two unstable equilibria of the pendulum on the surface, respectively. The velocity $d u / d t$ at the stable equilibrium of the system required to have the pendulum mass approach the unstable equilibrium asymptotically satisfies the energy equation

$$
\frac{m}{2}\left[\left.\frac{d u}{d t}\right|_{t=0}\right]^{2}=2 m|K| v^{2},
$$


i.e., the kinetic energy at $t=0$ is equal to the change in potential energy as $t \rightarrow \infty$. Solving for $d u / d t$ yields

$$
\left.\frac{d u}{d t}\right|_{t=0}=2 v \sqrt{|K|}, \quad \text { or }\left.\quad \frac{d \theta}{d t}\right|_{t=0}=v \sqrt{|K|} .
$$

This completes the proof of Theorem B.

Pendulum Conservation Law on Surfaces of Constant Curvature. Under the hypothesis of Theorem A, the following combination of intrinsic angular speed and position is constant:

$$
2\left(\frac{d \theta}{d t}\right)^{2}-K v^{2} \cos 2 \theta
$$

Proof. From Theorem A we have

$$
\frac{d^{2} \theta}{d t^{2}}=-K v^{2} \sin \theta \cos \theta=-\frac{K v^{2}}{2} \sin 2 \theta .
$$

We consider a change of variables as in the proof of Theorem A. If $K>0$, let $u=2 \theta$. If $K<0$, let $u=2 \theta+\pi$. In both cases the equation becomes

$$
\frac{d^{2} u}{d t^{2}}=-|K| v^{2} \sin u,
$$

which is the equation of a planar pendulum of length 1 in Euclidean space subject to a constant gravitational field of magnitude $g=|K| v^{2}$. The kinetic energy of this pendulum is $(m / 2)(d u / d t)^{2}$. Taking the potential energy to be zero when $u=\pi / 2$, the potential energy is $-m|K| v^{2} \cos u$. Their sum,

$$
\frac{m}{2}\left(\frac{d u}{d t}\right)^{2}-m|K| v^{2} \cos u,
$$

is constant. Changing back to $\theta$ and simplifying, this becomes

$$
2\left(\frac{d \theta}{d t}\right)^{2}-K v^{2} \cos 2 \theta
$$

\section{Proof of Theorem C.}

It should now be clear that the solutions of the constant speed pivot pendulums are symmetric with respect to the stable equilibria on a surface 
of constant curvature. Now we consider the case of the balanced barbell, such that the center of mass moves with constant speed along a geodesic. The symmetry of solutions for the pendulum imply that the masses can be treated independently and that if we do so, the solutions for the motion of the independent masses will lie at a fixed distance $\delta=d / 2$ from the pivot (which corresponds to the center of mass) and that a geodesic from one mass to the other will always pass through the pivot. This proves the theorem.

\section{Pendulum Motion for Accelerated Pivots}

\section{Proof of Theorem D.}

Now consider the case of pendulum motion on a surface of curvature $K$ when the pivot accelerates along a geodesic. Let $s(t)$ denote the arc-length parameter of the pivot at time $t$. When $K>0$, the equation of motion of the mass is then (c.f. proof of Theorem A)

$$
\begin{gathered}
\sigma(t)=R \sin _{K} \delta[\sin (\theta(t)) \hat{\mathbf{k}}+\cos (\theta(t))(-\sin (s(t) / R) \hat{\mathbf{i}}+\cos (s(t) / R) \hat{\mathbf{j}})] \\
+R \cos _{K} \delta(\cos (s(t) / R) \hat{\mathbf{i}}+\sin (s(t) / R) \hat{\mathbf{j}})
\end{gathered}
$$

where $R=1 / \sqrt{K}$.

As before, let $\left\{\mathbf{a}_{1}, \mathbf{a}_{2}\right\}$ be an oriented orthonormal frame for the tangent space of the surface at the point $A$ such that $\mathbf{a}_{2}$ is perpendicular to the rigid rod at the point $A$. We have

$$
\mathbf{a}_{2}(t)=\cos (\theta(t)) \hat{\mathbf{k}}-\sin (\theta(t))(-\sin (s(t) / R) \hat{\mathbf{i}}+\cos (s(t) / R) \hat{\mathbf{j}}) .
$$

Again, the force on the mass acts in the direction of the rod, and so $g\left(d^{2} \sigma / d t^{2}, \mathbf{a}_{2}(t)\right)=0$, which simplifies to

$$
R \sin _{K} \delta\left(\frac{d^{2} \theta}{d t^{2}}+\frac{v(t)^{2}}{R^{2}} \sin \theta(t) \cos \theta(t)\right)-a(t) \sin \theta(t) \cos _{K} \delta=0
$$

where $v(t)=d s / d t$ and $a(t)=d^{2} s / d t^{2}$ are the speed and scalar acceleration of the pivot. This can be rewritten as

$$
\frac{d^{2} \theta}{d t^{2}}=-K v(t)^{2} \cos \theta(t) \sin \theta(t)+\sqrt{K} a(t) \cot _{K} \delta \sin \theta(t)
$$


where $\cot _{K} \delta=\cos _{K} \delta / \sin _{K} \delta$. When $K<0$ the computation is similar, and one gets

$$
\frac{d^{2} \theta}{d t^{2}}=-K v(t)^{2} \cos \theta(t) \sin \theta(t)+\sqrt{-K} a(t) \cot _{K} \delta \sin \theta(t)
$$

and so the general formula is

$$
\frac{d^{2} \theta}{d t^{2}}=-K v(t)^{2} \cos \theta(t) \sin \theta(t)+\sqrt{|K|} a(t) \cot _{K} \delta \sin \theta(t) .
$$

The equilibria at $\theta=0, \pi$ are still present, and by linearizing one easily sees that they are still stable when $K>0$ and unstable when $K<0$. The other equilibria, however, are gone because the other angles for which $d^{2} \theta / d t^{2}=0$ now depend on $t$. This proves Theorem D.

The formula above should be compared with the formula in Theorem A. Note that if $a(t)=g$ and $K \rightarrow 0$, the equation reduces to the standard pendulum equation for constant acceleration,

$$
\frac{d^{2} \psi}{d t^{2}}+\frac{g}{\delta} \sin (\psi)=0
$$

where $\psi=\theta-\pi$.

For additional insight, assume that $s(t)=v_{0} t+a t^{2} / 2$, where $v_{0}$ and $a$ are constant. We have

$$
\frac{d^{2} \theta}{d t^{2}}=\left(-K\left(v_{0}+a t\right)^{2} \cos \theta(t)+\sqrt{|K|} a \cot _{K} \delta\right) \sin \theta(t) .
$$

When $K>0$, linearizing near $\theta=0$ yields

$$
\frac{d^{2} \theta}{d t^{2}} \approx\left(-K\left(v_{0}+a t\right)^{2}+\sqrt{K} a \cot _{K} \delta\right) \theta(t) .
$$

Linearizing near $\theta=\pi$ and replacing $\theta-\pi$ by $\psi$ yields

$$
\frac{d^{2} \psi}{d t^{2}} \approx\left(-K\left(v_{0}+a t\right)^{2}-\sqrt{K} a \cot _{K} \delta\right) \psi(t)
$$

These are similar to a pendulum in Euclidean space subject to an increasingly strong gravitational force. One surmises that the resulting oscillations have decreasing amplitude and period, something borne out by computer experiments. 
When $K<0$, we analyze the situation more heuristically. We have

$$
\frac{d^{2} \theta}{d t^{2}}=\left(-K\left(v_{0}+a t\right)^{2} \cos \theta(t)+\sqrt{-K} a \cot _{K} \delta\right) \sin \theta(t) .
$$

Let $\theta_{0}(t) \in[0, \pi]$ satisfy $-K\left(v_{0}+a t\right)^{2} \cos \theta_{0}(t)+\sqrt{-K} a \cot _{K} \delta=0$, which is possible for all sufficiently large $t$. We see that $\theta_{0}(t)$ is smooth and approaches $\pi / 2$ monotonically from above as $t \rightarrow \infty$. Assume that $\theta(t)$ is close to $\theta_{0}(t)$ and set $\psi(t)=\theta(t)-\theta_{0}(t)$. Assuming $t$ is large, we make the approximations $\sin \theta_{0}(t) \approx 1$ and $\theta_{0}^{\prime \prime}(t) \approx 0$. With these approximations, linearizing in $\psi$ yields

$$
\frac{d^{2} \psi}{d t^{2}} \approx K\left(v_{0}+a t\right)^{2} \psi
$$

This is similar to a pendulum in Euclidean space subject to an increasingly strong gravitational force. One surmises that the resulting oscillations have decreasing amplitude and period, and that the center of the oscillations tend toward $\pi / 2$ from above. This has also been observed empirically.

\section{References}

[1] Coulton, P., Galperin, G., Forces along equidistant particle paths, to appear in Mathematical Physics, Analysis and Geometry.

[2] Nagy, P., Dynamical invariants of rigid body motion on the hyperbolic plane, Geom. Dedicata, 37, 1991.

[3] Salvai M., On the dynamics of a rigid body in the hyperbolic space, Jour. of Geom. and Physics, 36, 2000.

[4] Zitterbarth, J., Some remarks on the motion of a rigid body in a space of constant curvature without external forces, Demonstratio Math., XXIV No. 3-4, 1991. 